\title{
A Compact Bias T Used for Front-end of Millimeter Imaging System
}

\author{
Baohua Yang ${ }^{1, a}$, Huajie Zou ${ }^{1, b}$ and Jungang Miao ${ }^{2, c}$ \\ ${ }^{1}$ Changzhou Vocational Institute of Mechatronic Technology, Changzhou, 213164, China; \\ ${ }^{2}$ School of Electronic and Information Engineering, Beihang University, Beijing, 100191, China. \\ ayangbaohua728@163.com, bzhj88000@163.com, cjmiaobremen@126.com
}

Keywords: bias T, triangle resonator, band-stop resonator, front-end.

\begin{abstract}
As millimeter wave (MMW) electronic technologies have matured, MMW receiver research is also in the rapid development which includes front-end and frequency synthesizer. They all needed isolation between RF and DC bias. The good design bias $\mathrm{T}$ which can realize high isolation between DC bias source port and RF ports is necessary. In this paper, two new reduced-sized broad-band resonators are introduced which can be used in the active circuits. Their bandwidths are controllable and the performance of one bias $\mathrm{T}$ is better than the same size of the compact bias $\mathrm{T}$ whose size reduction is $55.55 \%$ of the conventional radial stubs. Its improvement of S11, S12, and S13 is $18.8,8.2$, and $28.3 \%$ respectively. The performance of another kind bias $\mathrm{T}$ has a slightly inferior, but it has good stop-band characteristic at $2 \mathrm{GHz}$ and $8 \mathrm{GHz}$ where the $4 \mathrm{GHz}$ signal is needed. The two kinds of bias $\mathrm{T}$ perform as expected and the results are in good agreement with the simulated results, and have been used in the front-end circuit of our BHU-2D-U passive MMW imaging system.
\end{abstract}

\section{Introduction}

The human security inspection is an increasingly focus of many governments, its security, humanization and efficiency are raised with high requirement [1-3]. A series of $8 \mathrm{~mm}$ band two element instrument BHU-2D with disk antennas have been developed for application of human security apparatus by the Electromagnetics Laboratory of Beihang University [4].In the front-end circuit design of the BHU-2D-U system, a bias T for ultra wide band systems (UBW) could be used as a band-stop resonator when the isolation between the RF port and DC port is needed. The matching of the RF port should not be altered upon using a bias T[5], another important point to be considered is the bandwidth of the resonator, which should include the RF bandwidth, especially in the frequency synthesizer unite and LNA unite of BHU-2D-Ufront-end system, all signals should be rejected from signal ports to DC bias port that is the better RF choke and the RF signal should be passed between two RF ports with very lowly loss.

In order to achieve sufficient frequency bandwidth, several stubs are cascaded. One drawback of this method is its increasing size. Reza Dehbashi et al. have shown that a size impacted BRS whose size impaction ratio is about $55.55 \%$ and its shape is like to the Figure 2.[6].Triangle radial stub exhibits slightly higher radiation Q-factor than the corresponding circular micro-strip resonator [5]. This study introduces a new arrangement of triangle radial stub, whose bandwidth is controllable and the other parameters are better than the paper [5].

From the table I of section II, the improvement of S11, S12, and S13 is 18.8, 8.2, and 28.3\% respectively compare with the resonator of the paper [5] at the same size impaction. And then another new bias $\mathrm{T}$ is shown as Figure 3, it has the inferior effects as the resonators proposed by Reza Dehba shi et al., but it has notch effects at about $2 \mathrm{GHz}$ and $8 \mathrm{GHz}$.

The stop-band effects of the two designs would be changed with different radius A, and they are analyzed using ADS2008, the measurement results are agreement with theoretical results. 


\section{Analysis}

The isosceles triangle element configuration is shown in Fig. 1. It has an apex angle aand triangle side A. It can be analyzed by follows [7].

The TM-mode field patterns in a triangular-shaped are given by

$$
\left.\begin{array}{l}
E_{z}=A_{m, n, 1} T(x, y) \\
H_{x}=\frac{j}{\omega u_{0} u_{e}} \frac{\delta E_{z}}{\delta y} \\
H_{z}=\frac{-j}{\omega u_{0} u_{e}} \frac{\delta E_{z}}{\delta x} \\
H_{z}=E_{x}=E_{y}=0
\end{array}\right\}
$$

Where $A_{m, n, 1}$ is a constant. The complete standing wave solution is

$$
\begin{gathered}
E_{z}=A_{m, n, 1} T(x, y) \\
H_{x}=\frac{-j A_{m, n, 1}}{\omega u_{0} u_{e}}\left\{\begin{array}{l}
\left.\frac{2 \pi(m-n)}{3 A} \cos \left[\left(\frac{2 \pi x}{\sqrt{3} A}+\frac{2 \pi}{3}\right)\right]\right] \sin \left[\frac{2 \pi(m-n) y}{3 A}\right] \\
+\frac{2 \pi(n-1)}{3 A} \cos \left[\left(\frac{2 \pi x}{\sqrt{3} A}+\frac{2 \pi}{3}\right) m\right] \sin \left[\frac{2 \pi(n-1) y}{3 A}\right] \\
+\frac{2 \pi(1-m)}{3 A} \cos \left[\left(\frac{2 \pi x}{\sqrt{3} A}+\frac{2 \pi}{3}\right) n\right] \sin \left[\frac{2 \pi(1-m) y}{3 A}\right]
\end{array}\right\} \\
H_{y}=\frac{j A_{m, n, 1}}{\omega u_{0} u_{e}} \times\left\{\begin{array}{l}
\frac{2 \pi 1}{\sqrt{3} A} \sin \left[\left(\frac{2 \pi x}{\sqrt{3} A}+\frac{2 \pi}{3}\right) 1\right] \cos \left[\frac{2 \pi(m-n) y}{3 A}\right] \\
+\frac{2 \pi m}{3 A} \sin \left[\left(\frac{2 \pi x}{\sqrt{3} A}+\frac{2 \pi}{3}\right) m\right] \cos \left[\frac{2 \pi(n-1) y}{3 A}\right] \\
+\frac{2 \pi n}{\sqrt{3} A} \sin \left[\left(\frac{2 \pi x}{\sqrt{3} A}+\frac{2 \pi}{3}\right) n\right] \cos \left[\frac{2 \pi(1-m) y}{3 A}\right]
\end{array}\right\}
\end{gathered}
$$

Where $\mathrm{A}$ is the length of triangle side, $m+n+1=0$ and which satisfies the wave equation

$$
\begin{aligned}
& \left(\frac{\delta^{2}}{\delta x^{2}}+\frac{\delta^{2}}{\delta y^{2}}+k_{m, n, 1}^{2}\right) E_{2}=0 \\
& k_{m, n, 1}=\frac{4 \pi}{3 A} \sqrt{m^{2}+m n+n^{2}}
\end{aligned}
$$

The eigenvalue of the dominant mode $T M_{1,0,-1}$ and the neighbor order mode $T M_{1,1,-2}$ have been investigated.

As can be seen in Figure2 (b), it is the novel equivalent model for Figure 2(a).Figure2(c) is a novel bias $\mathrm{T}$ modified from Figure 2(b). The radius of resonators is all $5 \mathrm{~mm}$. In order to compare the two kinds of triangle resonators with the resonator proposed by the paper[5], the simulations are carried out with the substrate $\varepsilon_{r}=10.2$ and $h=1.27 \mathrm{~mm}$. From Figure 3 Figure 4 and Figure 5, these designs are analysed using ADS2008.

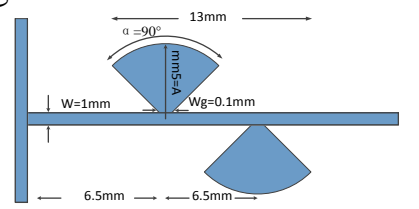

(a) The Bias $\mathrm{T}$ based on the cascaded BRS resonator

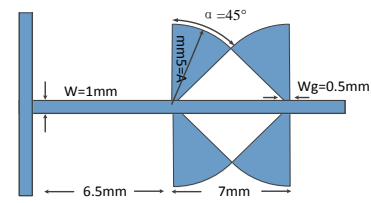

(b) Equivalent model bias $\mathrm{T}$ for (a) Fig1 The first bias $\mathrm{T}$ and its equivalent model. 


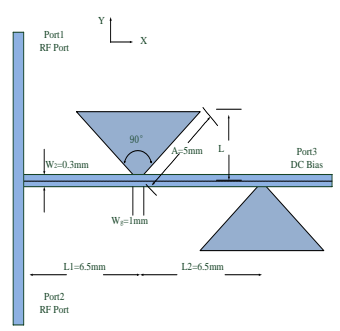

(a)The triangular Bias T

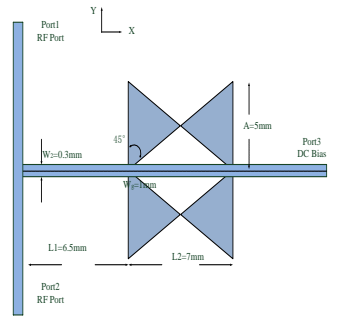

(b)Novel equivalent bias T1

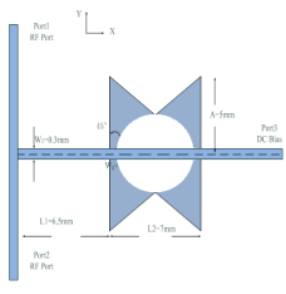

(c) A modified bias T2 from (b)

Fig2 The second bias $\mathrm{T}$ and its equivalent model.

From the simulation results at Figure3, Figure4 and Figure5, the triangle bias $\mathrm{T}$ has better effects than the paper [5] and the novel modified bias T has a slight inferior compared with the paper [5]. More details can be seen at the table I. The improvement of S11, S12, and S13 is 18.8, 8.2, and 28.3\% respectively compare with the resonator of the paper [5] at the same size impaction. For another new bias $\mathrm{T} 2$, the improvement of $\mathrm{S} 11, \mathrm{~S} 12$, and $\mathrm{S} 13$ is $-15.9,-6.1$, and $-14.5 \%$ respectively.

From Figure 1(a) and Figure2 (a) show a bias T using two radial stubs as a resonator. There are all three ports, port (1) and port(2) are RF inputs and outputs, port (3) is connected to the DC bias source. In order to isolate the RF ports and the DC bias port, a band-top filter is usually placed in between these ports which is typically cascaded radial stubs, such as Figure 1(a) and Figure2(a). They have the same parameters where $\mathrm{A}=5 \mathrm{~mm}, \alpha=90^{\circ}, L_{1}=L_{2}=6.5 \mathrm{~mm}$. A quarter of guided wavelength of mid-band frequency is required between the two radials and also the distance between the 1st radial and T junction, $w_{g}=1 \mathrm{~mm}, w_{1}=0.36 \mathrm{~mm}$ and $w_{2}=0.3 \mathrm{~mm}$ is the width of RF micro-strip line and DC feed line respectively. Figure 1(b) is the bias T proposed by Reza Dehbashi et al., and Figure2 (b) and Figure2(c) are our new bias $\mathrm{T}$ where $\mathrm{A}=5 \mathrm{~mm}, \alpha=45^{\circ}, L_{1}=6.5 \mathrm{~mm}$ and $L_{2}=7 \mathrm{~mm}$. The three kinds of bias $\mathrm{T}$ are the same parameters for comparing them conveniently.

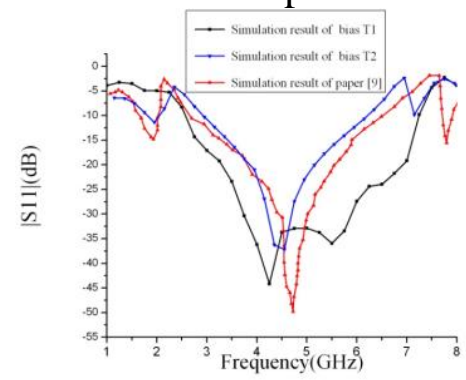

Fig3 Compared the simulation results of S11 among bias T1 and bias $\mathrm{T} 2$ and bias of paper[5]

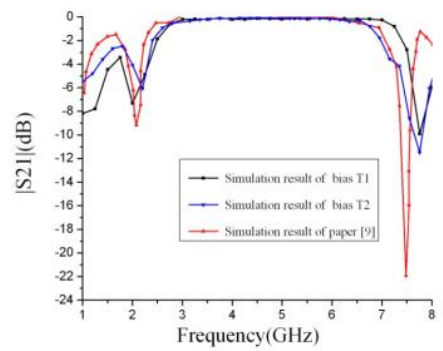

Fig4 Compared the simulation results of S12 among bias T1 and bias $\mathrm{T} 2$ and bias of paper[5]

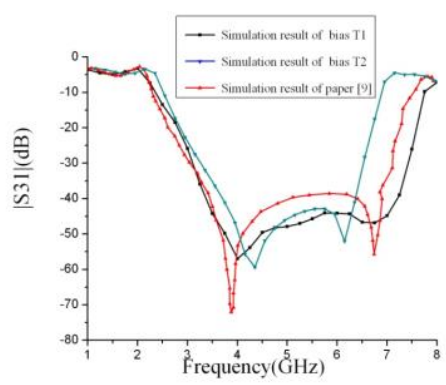

Fig5 Compared the simulation results of S31 among bias T1 and bias $\mathrm{T} 2$ and bias $\mathrm{T}$ of paper[5].

Table I Compared the simulation results among bias T1 and bias T2 and bias T of paper[5]

\begin{tabular}{|c|c|c|c|c|}
\hline \multicolumn{5}{|c|}{ Bandwidth(GHz) } \\
\hline S parameter & Bias T1 & Bias T2 & bias T of paper[5]. & $\begin{array}{c}\text { \%improvement } \\
\text { of T1 and T2 }\end{array}$ \\
\hline$|\mathrm{S} 11|>20 \mathrm{~dB}$ & $3.2-7.2$ & $3.8-5.1$ & $3.8-5.32$ & $28.3 ;-14.5$ \\
\hline $\mathrm{S} 12 \mid<3 \mathrm{~dB}$ & $2.4-7.7$ & $2.3-6.9$ & $2.3-7.2$ & $8.2 ;-6.1$ \\
\hline$|\mathrm{S} 31|<40 \mathrm{~dB}$ & $3.3-7.4$ & $3.6-6.5$ & $3.4-6.85$ & $18.8 ;-15.9$ \\
\hline
\end{tabular}

\section{Measurement}

From the Figure 6 (a),(b),(c)and(d), it shows that the radius of triangle resonator and modified triangle resonator vary from $3 \mathrm{~mm}$ to $5 \mathrm{~mm}$ by $0.5 \mathrm{~mm}$ steps. It consists of two couples of radial stubs on two sides of the transmission line. In this work, all radial stubs are placed in the middle of the transmission line, 
They are all realized on a ceramic filled soft substrate with dielectric constant of 6.2 and thickness of $0.254 \mathrm{~mm}$ adopted by the front end circuit of the BHU-2D system[4]. Their measurement results are shown as Figure 7 and Figure 8 , the $\mathrm{S} 21$ of Figure 7 is less than $-10 \mathrm{~dB}$ from $4 \mathrm{GHz}$ to $8 \mathrm{GHz}$ as A from $5 \mathrm{~mm}$ to $3.5 \mathrm{~mm}$ in Figure7 and the S21 of Figure 8 from $5 \mathrm{~mm} 3 \mathrm{~mm}$. It shows that the modified resonator has the more bandwidth for bandstop.

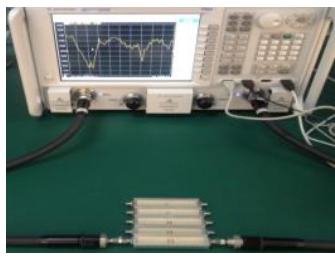

(a) Measurement for triangle resonator

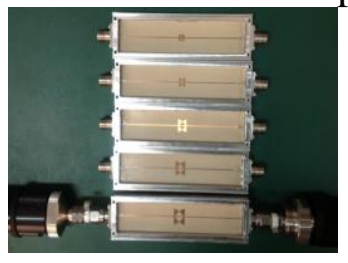

(b) Series of triangle resonator

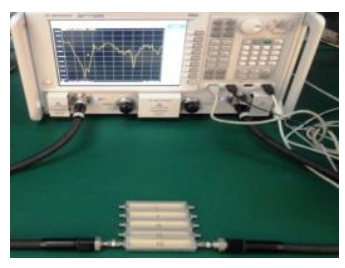

(c)Measurement for modified triangle resonator

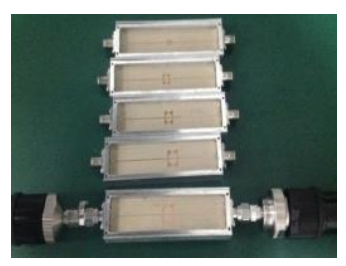

(d) Series of modified triangle resonator

Fig 6 Measurement of the novel triangle resonator

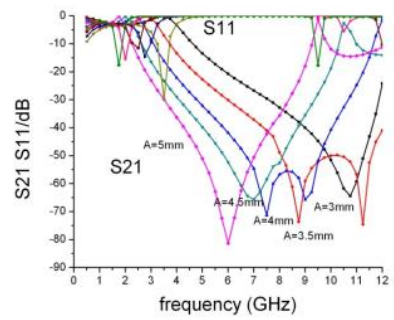

Fig 7 Frequency response of the novel triangle resonator

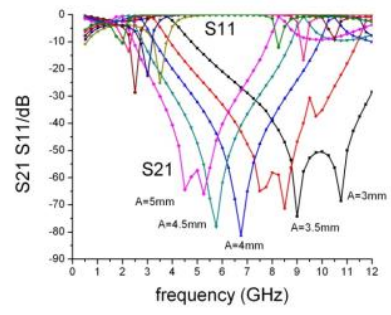

Fig 8 Frequency response of the modified triangle resonator

Table II Compared the measurement results betweenthe novel triangle resonator and the modified triangle resonator

\begin{tabular}{|c|c|c|c|c|c|c|}
\hline \multicolumn{5}{|c|}{ Bandwidth(GHz) } & & \\
\hline Radius A(mm) & & 5 & 4.5 & 4 & 3.5 & 3 \\
\hline \multirow{2}{*}{$|\mathrm{S} 11|<3 \mathrm{~dB}$} & 7.25 & 7.5 & 8.25 & 9.1 & 12 & 7.25 \\
\cline { 2 - 7 } & 6.75 & 6.5 & 8.5 & 10.5 & 11.4 & 6.75 \\
\hline \multirow{2}{*}{$|\mathrm{S} 21|>40 \mathrm{~dB}$} & 3 & 3.5 & 4.25 & 5.2 & 5.85 & 3 \\
\cline { 2 - 7 } & 2 & 2.25 & 2.3 & 2.5 & 3.2 & 2 \\
\hline
\end{tabular}

From the table II, the radius of the novel triangle resonator and the modified triangle resonator are all changed from $5 \mathrm{~mm}$ to $3 \mathrm{~mm}$ by step of $0.5 \mathrm{~mm}$. According to the active design of system, two bias $\mathrm{T}$ are designed at Figure9 (a) and (b).

From Figure 9 (a) and (b), it shows the triangle bias $\mathrm{T}$ and the modified triangle bias $\mathrm{T}$ respectively. The space shape between two triangle resonator at either side of DC feed line is changed as semicircle for Figure9 (b). According to the requirement of active circuit, the signal of $2 \mathrm{GHz}, 4 \mathrm{GHz}$ and $8 \mathrm{GHz}$ should be rejected from signal ports to DC bias port that is the better RF choke, the signal of $4 \mathrm{GHz}$ should be passed between two RF ports. Because there are $2 \mathrm{GHz}$ and $8 \mathrm{GHz}$ signals among the output signal at $4 \mathrm{GHz}$ which come from frequency multiplier unite or LNA unite. The $\mathrm{S}$ parameters of the circuits were measured using a N5225A Agilent network analyser. The results are shown in Figures 10, 11 and 12 respectively. The $\mathrm{S} 11$ of the two bias $\mathrm{T}$ are below $-10 \mathrm{~dB}$ at $2 \mathrm{GHz}, 4 \mathrm{GHz}$ and $8 \mathrm{GHz}$, the $\mathrm{S} 21$ the two bias are near $0.1 \mathrm{~dB}$ at RF signal $4 \mathrm{GHz}$ and only the $\mathrm{S} 21$ of the modified triangle bias $\mathrm{T}$ are below $-10 \mathrm{~dB}$ at $2 \mathrm{GHz}$ and $8 \mathrm{GHz}$. The $\mathrm{S} 31$ of two bias T are below $-15 \mathrm{~dB}$ at $2 \mathrm{GHz}, 4 \mathrm{GHz}$ and $8 \mathrm{GHz}$. It can be seen that circuits perform as expected and the results are in good agreement with the simulated results. 

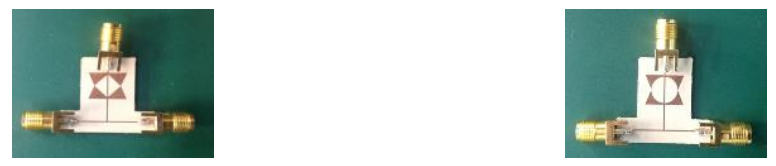

$\begin{array}{lll}\text { (a) the novel triangle bias } \mathrm{T} 1 & \text { (b) the modified triangle bias } \mathrm{T} 2\end{array}$

Fig9 The two bias T1 and T2

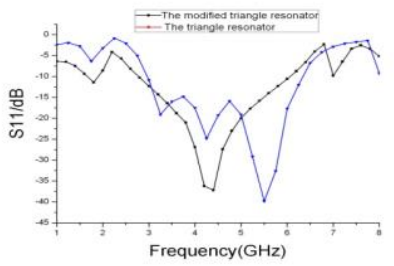

Fig 10 The S11 of T1 and T2

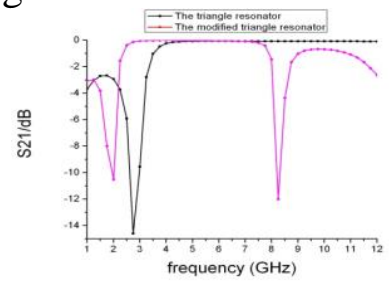

Fig 11 The S21 of T1 and T2

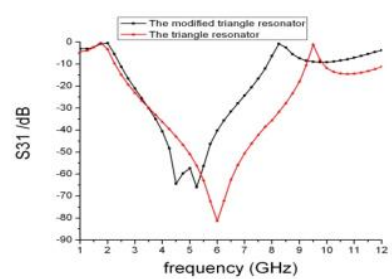

Fig $12 \mathrm{The} \mathrm{S} 31$ of $\mathrm{T} 1$ and $\mathrm{T} 2$

\section{Summary}

In this work, two novel compact broad-band band-stop resonators are presented. The resonator's size is reduced to about $55.55 \%$ of a conventional two radial stub resonators. Its bandwidth can be controllable and has better performance. The two circuits perform as expected and the results are in good agreement with the simulated results. It is shown that the two resonators can be used in applications as bias T circuits of front-end of MMW design. According to the front-end design of MMW more broad-band bias $\mathrm{T}$ and band-stop resonators will be investigated in the future work.

\section{Acknowledgments}

This work was supported by the Natural Science youth fund of Jiangsu Province, China under Grant BK20160296.

\section{References}

[1]. Li T, Jin W, Zhao G. Processing and fusion of passively acquired, millimeter and terahertz images of the human body[J]. Optical Engineering, 2017, 56(4):043102.

[2]. Mohammadzade H, Ghojogh B, Faezi S, et al. Critical object recognition in millimeter-wave images with robustness to rotation and scale.[J]. J Opt Soc Am A Opt Image Sci Vis, 2017, 34(6):846-855.

[3]. Mohammadzade H, Ghojogh B, Faezi S, et al. Critical object recognition in millimeter-wave images with robustness to rotation and scale.[J]. J Opt Soc Am A Opt Image Sci Vis, 2017, 34(6):846-855.

[4]. Bao-Hua Yang, Ghulam Mehdi, Anyong Hu, etc., "THE ROUND-ENDED DESIGN AND MEASUREMENT OF ALL SYMMETRIC EDGE-COUPLED BANDPASS FILTER", Progress In Electromagnetics Research, Vol. 136, 2013, pp:17-27.

[5]. Reza Dehbashi, Hamidreza Dalili Oskouei, and Keyvan Forooraghi, "A NOVEL BROAD-BAND MICROSTRIPRADIAL STUB RESONATOR USED INBIAS-T APPLICATION", MICROWAVE AND OPTICAL TECHNOLOGY LETTERS / Vol. 48, No. 9, September 2006, 1766-1770.

[6]. ARVIND K. SHARMA,"Analysis of Triangular MIcrostrip Resonators", IEEETRANSAC $\sim$ ONSON MICROWAVETREORYAND TECHNIQUESVOL. $30, \quad$ NO. 11 , NOVS14SER1982 2029-2031

[7]. JOSEPH HELSZAJN,“ Planar Triangular "Resonators with Magnetic Walk", IEEE TRANSACTIONS ON MICROWAVE THEORY AND TECHNIQ(JES, VOL. MTT-26, NO. 2, FEBRUARY 1978 